\title{
Second Hankel Determinants for the Class of Typically Real Functions
}

\author{
Pawel Zaprawa \\ Department of Mathematics, Lublin University of Technology, Nadbystrzycka 38D, 20-618 Lublin, Poland \\ Correspondence should be addressed to Paweł Zaprawa; p.zaprawa@pollub.pl
}

Received 5 November 2015; Revised 22 December 2015; Accepted 3 January 2016

Academic Editor: Marco Donatelli

Copyright (C) 2016 Paweł Zaprawa. This is an open access article distributed under the Creative Commons Attribution License, which permits unrestricted use, distribution, and reproduction in any medium, provided the original work is properly cited.

We discuss the Hankel determinants $H_{2}(n)=a_{n} a_{n+2}-\left(a_{n+1}\right)^{2}$ for typically real functions, that is, analytic functions which satisfy the condition $\operatorname{Im} z \operatorname{Im} f(z) \geq 0$ in the unit disk $\Delta$. Main results are concerned with $H_{2}(2)$ and $H_{2}(3)$. The sharp upper and lower bounds are given. In general case, for $n \geq 4$, the results are not sharp. Moreover, we present some remarks connected with typically real odd functions.

\section{Introduction}

Let $\Delta$ be the unit disk $\{z \in \mathbb{C}:|z|<1\}$ and let $\mathscr{A}$ be the family of all functions $f$ analytic in $\Delta$ that have the Taylor series expansion $f(z)=z+\sum_{n=2}^{\infty} a_{n} z^{n}$. In $[1,2]$ Pommerenke defined $q$ th Hankel determinant for a function $f$ as

$$
H_{q}(n)=\left|\begin{array}{cccc}
a_{n} & a_{n+1} & \cdots & a_{n+q-1} \\
a_{n+1} & a_{n+2} & \cdots & a_{n+q} \\
\cdots & \cdots & \cdots & \cdots \\
a_{n+q-1} & a_{n+q} & \cdots & a_{n+2 q-2}
\end{array}\right|,
$$

where $n, q \in \mathbb{N}$.

Recently, the Hankel determinant has been studied intensively by many mathematicians. The research was focused on $\mathrm{H}_{2}$ (2) for various classes of univalent functions. The papers by Janteng et al. [3, 4], Lee et al. [5], Vamshee Krishna and Ramreddy [6], and Selvaraj and Kumar [7] are worth mentioning here. Janteng et al. derived the exact bounds of $\left|H_{2}(2)\right|$ for the classes: $S^{*}$ of star-like functions $\left(\left|H_{2}(2)\right| \leq 1\right)$, $\mathscr{K}$ of convex functions $\left(\left|H_{2}(2)\right| \leq 1 / 8\right)$, and $\mathscr{R}$ of functions whose derivative has a positive real part $\left(\left|H_{2}(2)\right| \leq 4 / 9\right)$. Lee et al. [5] investigated the Hankel determinant in the general class $S^{*}(\varphi)$ of star-like functions with respect to a given function $\varphi$. This class was defined by Ma and Minda in [8]. In particular, Lee et al. obtained the results for the following classes: $S^{*}(\alpha)$ of star-like functions of order $\alpha\left(\left|H_{2}(2)\right| \leq(1-\right.$ $\left.\alpha)^{2}\right), \mathcal{S} \mathscr{L}^{*}$ of lemniscate star-like functions $\left(\left|H_{2}(2)\right| \leq 1 / 16\right.$; for the definition of $\mathcal{S} \mathscr{L}^{*}$, see [9]), and $S_{\beta}^{*}$ of strongly starlike functions of order $\beta\left(\left|H_{2}(2)\right| \leq \beta^{2}\right)$. Vamshee Krishna and Ramreddy [6] generalized the result of Janteng et al. They gave the bound of $\left|H_{2}(2)\right|$ in the class $\mathscr{K}(\alpha)$ of convex functions of order $\alpha$. Selvaraj and Kumar [7] proved that the estimate of the second Hankel determinant for the class $\mathscr{C}$ of close-to-convex functions is the same as that for the class $S^{*}$. The question whether this bound is good for the class $S$ of all univalent functions has no answer yet. One can find some other results in this direction in [10-14].

Taking different set of parameters $q$ and $n$, the research on the Hankel determinant is much more difficult. In [15] Hayami and Owa discussed $\mathrm{H}_{2}(n)$ for functions $f$ satisfying $\operatorname{Re} f(z) / z>\alpha$ or $\operatorname{Re} f^{\prime}(z)>\alpha$. On the other hand, Babalola [16] tried to estimate $\left|H_{3}(1)\right|$ for $S^{*}, \mathscr{K}$, and $\mathscr{R}$. Shanmugam et al. [17] discussed $\left|H_{3}(1)\right|$ for the class $M_{\alpha}$ of $\alpha$-star-like functions defined by Mocanu in [18].

In particular, if $q=2$ and $n=1$ then $H_{2}(1)$ is known as a classical functional of Fekete-Szegö. A lot of papers have been devoted to the studies concerning this functional. Because $\mathrm{H}_{2}(1)$ is not related to the subject of this paper, we omit recalling results obtained in this direction.

The majority of results concerning the Hankel determinants were obtained for univalent functions. In this paper we discuss functions which, in general, are not univalent. We focus our investigation on typically real functions. 


\section{Class $T$ and the Hankel Determinants for a Selected Functions in $T$}

A function $f \in \mathscr{A}$ that satisfies the condition $\operatorname{Im} z \operatorname{Im} f(z) \geq$ 0 for $z \in \Delta$ is called a typically real function. Let $T$ denote the class of all typically real functions. Robertson [19] proved that $f \in T$ if and only if there exists a probability measure $\mu$ on $[-1,1]$ such that the following formula holds:

$$
f(z)=\int_{-1}^{1} \frac{z}{1-2 z t+z^{2}} d \mu(t)
$$

The coefficients of a function $f(z)=z+\sum_{n=2}^{\infty} a_{n} z^{n} \in T$ can be written as follows:

$$
\begin{array}{r}
a_{n}=\int_{-1}^{1} \frac{\sin (n \arccos t)}{\sin (\arccos t)} d \mu(t)=\int_{-1}^{1} U_{n-1}(t) d \mu(t), \\
n=1,2, \ldots
\end{array}
$$

The functions $U_{n}(t), n=1,2, \ldots$, which appear in the above formula, are the well-known Chebyshev polynomials of the second kind.

Since all coefficients of $f \in T$ are real we look for the lower and the upper bounds of $\mathrm{H}_{2}(2)$ instead of the bound of $\left|H_{2}(2)\right|$. At the beginning, let us look at a few examples.

Example 1. All the functions $f_{t}(z)=z /\left(1-2 z t+z^{2}\right), t \in$ $[-1,1]$, are in $S^{*}$. Since $f_{t}(z)=z+2 t z^{2}+\left(4 t^{2}-1\right) z^{3}+\left(8 t^{3}-\right.$ $4 t) z^{4}+\cdots$, we have $H_{2}(2)=-1$ for each $t \in[-1,1]$. Moreover, $H_{2}(n)=U_{n-1}(t) U_{n+1}(t)-U_{n}(t)^{2}$. This and the Turan identity for Chebyshev polynomials $U_{n}(t)$ result in $H_{2}(n)=-1$ for each $n=2,3, \ldots$.

Example 2. For a function $f(z)=z\left(1+z^{2}\right) /\left(1-z^{2}\right)^{2}$ having the Taylor series expansion $f(z)=z+3 z^{3}+5 z^{5}+\cdots$ there is $H_{2}(n)=-(n+1)^{2}$ for even $n$ and $H_{2}(n)=(n+1)^{2}-1$ for odd $n$. In this case, the function $f$ is not univalent; the bound of $\left|H_{2}(2)\right|$ is much greater than 1 , the value of the second Hankel determinant for star-like functions or close-to-convex functions.

Example 3. Every Hankel determinant $H_{2}(n), n=1,2, \ldots$, for a function $f(z)=\log (1 /(1-z))=z+(1 / 2) z^{2}+(1 / 3) z^{3}+\cdots$ is positive. Namely, $H_{2}(n)=1 / n(n+1)^{2}(n+2)$.

For a given class $A \subset \mathscr{A}$, we denote by $\Omega_{n}(A), n \geq 1$, the region of variability of three succeeding coefficients of functions in $A$, that is, the set $\left\{\left(a_{n}(f), a_{n+1}(f), a_{n+2}(f)\right)\right.$ : $f \in A\}$. As it is seen in (3), the coefficients of typically real functions are the Stieltjes integrals of the Chebyshev polynomials of the second kind with respect to a probability measure. Hence, $\Omega_{n}(T)$ is the closed convex hull of the curve $\gamma:[-1,1] \ni t \rightarrow\left(U_{n-1}(t), U_{n}(t), U_{n+1}(t)\right)$ (see, e.g., [20]).

Lemma 4. The functional $T \ni f \rightarrow H_{2}(n), n \geq 2$, attains its extreme values on the boundary of $\Omega_{n}(T)$.
Proof. The only critical point of $h(x, y, z)=x z-y^{2}$, where $x=a_{n}, y=a_{n+1}$, and $z=a_{n+2}$, is $(0,0,0)$. But $h(0,0,0)=0$. Since $h$ may be positive as well as negative for $(x, y, z) \epsilon$ $\Omega_{n}(T)$, (see Examples 1 and 3 ), it means that the extreme values of $h$ are attained on the boundary of $\Omega_{n}(T)$.

\section{Bounds of $H_{2}(2)$ in $T$}

In [21] Ma proved so-called generalized Zalcman conjecture for the class $T$ :

$$
\begin{array}{ll} 
& \left|a_{n} a_{m}-a_{n+m-1}\right| \\
\quad \leq \begin{cases}n+1, & m=2, n=2,4,6, \ldots, \\
m+1, & n=2, m=2,4,6, \ldots, \\
(n-1)(m-1), & \text { otherwise. }\end{cases}
\end{array}
$$

We apply this result to prove the following.

Theorem 5. If $f \in T$ then $\left|H_{2}(2)\right| \leq 9$.

Proof. The result of $\mathrm{Ma}$ and the triangle inequality result in

$$
\left|a_{2} a_{4}-a_{3}^{2}\right| \leq\left|a_{2} a_{4}-a_{5}\right|+\left|a_{5}-a_{3}^{2}\right| \leq 5+4=9 .
$$

This result is sharp; the equality holds for $f(z)=z(1+$ $\left.z^{2}\right) /\left(1-z^{2}\right)^{2}$. Furthermore, we can see the following.

Corollary 6. For $T$ one has

$$
\min \left\{H_{2}(2): f \in T\right\}=-9 .
$$

For our next theorem let us cite two results. First one is the obvious conclusion from the Carathéodory theorem and the Krein-Milman theorem. We assume that $X$ is a compact Hausdorff space and

$$
J_{\mu}=\int_{X} J(t) d \mu(t)
$$

Theorem A (see [22, Thm. 1.40]). If J :X $\rightarrow \mathbb{R}^{n}$ is continuous then the convex hull of $J(X)$ is a compact set and it coincides with the set $\left\{J_{\mu}: \mu \in P_{X},|\operatorname{supp}(\mu)| \leq n\right\}$.

In the above, the symbols $P_{X}$ and $|\operatorname{supp}(\mu)|$ stand for the set of probability measures on $X$ and the cardinality of the support of $\mu$, respectively.

It means that $\mu$ is atomic measure having at most $n$ steps. More precise information about the relation between the measure and the convex hull is presented in the following theorem. In what follows, $\langle\vec{a}, \vec{b}\rangle$ means the scalar product of $\vec{a}$ and $\vec{b}$.

Theorem B (see [22, Thm. 1.49]). Let $J:[\alpha, \beta] \rightarrow \mathbb{R}^{n}$ be continuous. Suppose that there exists a positive integer $k$, such that for each nonzero $p$ in $\mathbb{R}^{n}$ the number of solutions of any equation $\langle\overrightarrow{J(t)}, \vec{p}\rangle=$ const, $\alpha \leq t \leq \beta$, is not greater than $k$. 
Then, for every $\mu \in P_{[\alpha, \beta]}$ such that $J_{\mu}$ belongs to the boundary of the convex hull of $J([\alpha, \beta])$ the following statements are true:

(1) If $k=2 m$ then

(a) $|\operatorname{supp}(\mu)| \leq m$, or

(b) $|\operatorname{supp}(\mu)|=m+1$ and $\{\alpha, \beta\} \subset \operatorname{supp}(\mu)$.

(2) If $k=2 m+1$ then

(a) $|\operatorname{supp}(\mu)| \leq m$, or

(b) $|\operatorname{supp}(\mu)|=m+1$ and one of the points $\alpha$ and $\beta$ belongs to $\operatorname{supp}(\mu)$.

This theorem, in slightly modified version, was published in [23] as Lemma 2.

Putting $J(t)=\left[U_{1}(t), U_{2}(t), U_{3}(t)\right], t \in[-1,1]$, and $\vec{p}=$ $\left[p_{1}, p_{2}, p_{3}\right]$, we can see that any equation of the form

$$
p_{1} U_{1}(t)+p_{2} U_{2}(t)+p_{3} U_{3}(t)=\text { const }, \quad t \in[-1,1]
$$

is equivalent to $W_{3}(t)=$ const, where $W_{3}(t)$ is a polynomial of degree 3 . Hence, (8) has at most 3 solutions. According to Theorem $\mathrm{B}$, the boundary of the convex hull of $J([-1,1])$ is determined by atomic measures $\mu$ for which support consists of at most 2 points. Moreover, one of them has to be -1 or 1 . We have proved the following.

Lemma 7. The boundary of $\Omega_{2}(T)$ consists of points $\left(a_{2}, a_{3}, a_{4}\right)$ that correspond to the following functions:

$$
\begin{aligned}
f(z)=\alpha \frac{z}{1-2 z t+z^{2}}+(1-\alpha) & \frac{z}{(1-z)^{2}}, \\
& \alpha \in[0,1], t \in[-1,1]
\end{aligned}
$$

or

$$
\begin{aligned}
f(z)=\alpha \frac{z}{1-2 z t+z^{2}}+(1-\alpha) & \frac{z}{(1+z)^{2}}, \\
& \alpha \in[0,1], t \in[-1,1] .
\end{aligned}
$$

Now, we are ready to prove the following.

Theorem 8. For $T$ one has

$$
\max \left\{H_{2}(2): f \in T\right\}=1
$$

Proof. By Lemma 7, it is enough to take functions given by (9) or (10). Consider the following:

(I) Function (9) has the series expansion

$$
\begin{aligned}
f(z)= & +2(1-\alpha+\alpha t) z^{2} \\
& +\left[3(1-\alpha)+\left(4 t^{2}-1\right) \alpha\right] z^{3} \\
& +4\left[1-\alpha+\left(2 t^{3}-t\right) \alpha\right] z^{4}+\cdots
\end{aligned}
$$

Hence, $H_{2}(2)=g_{1}(\alpha, t)$, where

$$
\begin{aligned}
g_{1}(\alpha, t)= & 8(1-\alpha+\alpha t)\left[1-\alpha+\left(2 t^{3}-t\right) \alpha\right] \\
& -\left(4 t^{2} \alpha-4 \alpha+3\right)^{2}
\end{aligned}
$$

$\alpha \in[0,1], t \in[-1,1]$.

From

$$
\begin{aligned}
& \frac{\partial g_{1}}{\partial \alpha}=8\left(1-t^{2}\right)\left(2(2-\alpha) t^{2}+1-2 \alpha\right), \\
& \frac{\partial g_{1}}{\partial t}=16 \alpha(\alpha-1)\left(4 t^{2}-3 t+3\right),
\end{aligned}
$$

it follows that the critical points of $g_{1}$ are as follows: $(0,-1)$, $(0,1),(1,-1),(1,1),(1,-1 / \sqrt{2}),(1,1 / \sqrt{2})$, and $(1 / 2,0)$. Among these points, only $(1 / 2,0)$ lies inside the set $[0,1] \times$ $[-1,1]$.

If $\alpha=0$ or $\alpha=1$ then functions (9) coincide with $f_{t}$ from Example 1. If $t=1$ then $f(z)=z /(1-z)^{2}$. In each case $H_{2}(2)=-1$. For $t=-1$, function (9) takes the form $f(z)=\alpha\left(z /(1+z)^{2}\right)+(1-\alpha)\left(z /(1-z)^{2}\right)$. Then $H_{2}(2)=$ $8(1-2 \alpha)^{2}-9 \leq-1$.

If $\alpha=1 / 2$ and $t=0$ we have $H_{2}(2)=1$. It means that the greatest value of $\mathrm{H}_{2}$ (2) for functions given by (9) is equal to 1 . The extremal function is

$$
\begin{aligned}
f(z) & =\frac{1}{2}\left[\frac{z}{1+z^{2}}+\frac{z}{(1-z)^{2}}\right] \\
& =z+z^{2}+z^{3}+2 z^{4}+3 z^{5}+\cdots
\end{aligned}
$$

(II) For functions (10), $H_{2}(2)$ is equal to $g_{2}(\alpha, t)$, where

$$
\begin{aligned}
g_{2}(\alpha, t)= & 8(-1+\alpha+\alpha t)\left[-1+\alpha+\left(2 t^{3}-t\right) \alpha\right] \\
& -\left(4 t^{2} \alpha-4 \alpha+3\right)^{2}
\end{aligned}
$$

$$
\alpha \in[0,1], t \in[-1,1] .
$$

Moreover, $g_{2}(\alpha, t)=g_{1}(\alpha,-t)$. Taking into account the symmetry of the range of variability of $t$, we obtain the same result as above also for functions defined by (10). The extremal function is

$$
\begin{aligned}
f(z) & =\frac{1}{2}\left[\frac{z}{1+z^{2}}+\frac{z}{(1+z)^{2}}\right] \\
& =z-z^{2}+z^{3}-2 z^{4}+3 z^{5}+\cdots
\end{aligned}
$$

\section{Bounds of $H_{2}(3)$ in $T$}

The proof of the following theorem is obvious.

Theorem 9. If $n$ is odd then

$$
\max \left\{H_{2}(n): f \in T\right\}=n(n+2) .
$$

Hence, one has the following. 
Corollary 10. For $T$ one has

$$
\max \left\{H_{2}(3): f \in T\right\}=15 .
$$

In similar way, as it was done for Lemma 7, one can prove the following.

Lemma 11. The boundary of $\Omega_{3}(T)$ consists of points $\left(a_{3}\right.$, $a_{4}, a_{5}$ ) that correspond to the following functions:

$$
\begin{aligned}
f(z)=\alpha \frac{z}{1-2 z t_{1}+z^{2}}+(1-\alpha) \frac{z}{1-2 z t_{2}+z^{2}}, \\
\quad \alpha \in[0,1], t_{1}, t_{2} \in[-1,1]
\end{aligned}
$$

or

$$
\begin{aligned}
& f(z)= \alpha \frac{z}{(1+z)^{2}}+\beta \frac{z}{(1-z)^{2}} \\
&+(1-\alpha-\beta) \frac{z}{1-2 z t+z^{2}}, \\
& \quad \alpha, \beta \in[0,1], \alpha+\beta \leq 1, t \in[-1,1] .
\end{aligned}
$$

Theorem 12. For $T$ one has

$$
\min \left\{H_{2}(3): f \in T\right\}=-\frac{4}{3}+\frac{8}{9} \sqrt{6}=-3.51 \ldots
$$

Proof. By Lemma 11, it suffices to discuss functions given by (20) or (21). Consider the following:

(I) For functions (20), we have

$$
\begin{aligned}
a_{3}= & \left(4 t_{1}^{2}-1\right) \alpha+\left(4 t_{2}^{2}-1\right)(1-\alpha), \\
a_{4}= & \left(8 t_{1}^{3}-4 t_{1}\right) \alpha+\left(8 t_{2}^{3}-4 t_{2}\right)(1-\alpha), \\
a_{5}= & \left(16 t_{1}^{4}-12 t_{1}^{2}+1\right) \alpha \\
& +\left(16 t_{2}^{4}-12 t_{2}^{2}+1\right)(1-\alpha),
\end{aligned}
$$

and, hence, applying the Turan identity, $H_{2}(3)=g_{3}\left(\alpha, t_{1}, t_{2}\right)$, where

$$
\begin{aligned}
& g_{3}\left(\alpha, t_{1}, t_{2}\right) \\
& =-\alpha^{2}-(1-\alpha)^{2} \\
& +2 \alpha(1-\alpha)\left[8\left(t_{1}-t_{2}\right)^{2}\left(1-t_{1}^{2}\right)\left(1-t_{2}^{2}\right)-1\right], \\
& \quad \alpha \in[0,1], t_{1}, t_{2} \in[-1,1] .
\end{aligned}
$$

The expression in brackets is greater than or equal to -1 for all $t_{1}, t_{2} \in[-1,1]$. Hence,

$$
H_{2}(3) \geq-\alpha^{2}-(1-\alpha)^{2}-2 \alpha(1-\alpha)=-1 \text {. }
$$

(II) If function $f$ is given by (21) then

$$
\begin{aligned}
& a_{3}=3(\alpha+\beta)+\left(4 t^{2}-1\right)(1-\alpha-\beta), \\
& a_{4}=4(\beta-\alpha)+\left(8 t^{3}-4 t\right)(1-\alpha-\beta), \\
& a_{5}=5(\alpha+\beta)+\left(16 t^{4}-12 t^{2}+1\right)(1-\alpha-\beta) .
\end{aligned}
$$

Using the Turan identity, it follows that $H_{2}(3)=g_{4}(\alpha, \beta, t)$, where

$$
\begin{aligned}
g_{4}(\alpha, \beta, t)= & -1+2(\alpha+\beta)-2(\alpha+\beta)^{2}+64 \alpha \beta \\
& +2(1-\alpha-\beta) q(t), \\
q(t)= & (\alpha+\beta)\left(24 t^{4}-8 t^{2}-1\right) \\
& +(\alpha-\beta)\left(32 t^{3}-16 t\right),
\end{aligned}
$$

under the assumptions $\alpha, \beta \in[0,1], \alpha+\beta \leq 1$, and $t \in[-1,1]$. Let $\alpha$ and $\beta$ be fixed. Since

$$
\frac{\partial q}{\partial t}=8\left(6 t^{2}-1\right)[t(\alpha+\beta)+\alpha-\beta]
$$

the critical points of $q$ are as follows: $-1 / \sqrt{6}, 1 / \sqrt{6}$, and $(\beta-$ $\alpha) /(\alpha+\beta)$. It is easily seen that all these points are in $[-1,1]$. Therefore,

$$
\begin{aligned}
& \min \{q(t): t \in[-1,1]\}=\min \left\{q(-1), q\left(\frac{-1}{\sqrt{6}}\right),\right. \\
& \left.q\left(\frac{1}{\sqrt{6}}\right), q(1), q\left(\frac{\beta-\alpha}{\alpha+\beta}\right)\right\} .
\end{aligned}
$$
form

For $t=-1$ or $t=1$, the functions given by (21) have the

$$
f(z)=\widetilde{\alpha} \frac{z}{(1+z)^{2}}+\widetilde{\beta} \frac{z}{(1-z)^{2}}, \quad \widetilde{\alpha}+\widetilde{\beta}=1 .
$$

One can show directly from formula (30) that

$$
H_{2}(3)=-1+64 \widetilde{\alpha}(1-\widetilde{\alpha}) \geq-1 .
$$

For $t=(\beta-\alpha) /(\alpha+\beta)$, there is

$$
H_{2}(3)=-1+\frac{64 \alpha \beta\left[4(\alpha+\beta) \alpha \beta+(\beta-\alpha)^{2}\right]}{(\alpha+\beta)^{3}} \text {; }
$$

hence,

$$
H_{2}(3) \geq-1
$$

If $t=-1 / \sqrt{6}$ or $t=1 / \sqrt{6}$ then $H_{2}$ (3) is equal to

$$
\begin{aligned}
& H_{2}(3) \\
& =-1+2(\alpha+\beta)-2(\alpha+\beta)^{2}+64 \alpha \beta \\
& +(1-\alpha-\beta)\left[-\frac{10}{3}(\alpha+\beta)-\frac{64}{3 \sqrt{6}}(\alpha-\beta)\right]
\end{aligned}
$$

or

$$
\begin{aligned}
& H_{2}(3) \\
& =-1+2(\alpha+\beta)-2(\alpha+\beta)^{2}+64 \alpha \beta \\
& +(1-\alpha-\beta)\left[-\frac{10}{3}(\alpha+\beta)+\frac{64}{3 \sqrt{6}}(\alpha-\beta)\right],
\end{aligned}
$$


respectively. Without loss of generality, we can assume that $\alpha \geq \beta$. Then, while looking for the minimum value of $\mathrm{H}_{2}(3)$, we can restrict the research to the first stated above case (since expression (35) is not less than expression (34)).

Transforming (35), we obtain

$$
\begin{aligned}
H_{2}(3)= & -1+64 \alpha \beta-\frac{4}{3}(\alpha+\beta)(1-\alpha-\beta) \\
& +\frac{64}{3 \sqrt{6}}(\beta-\alpha)(1-\alpha-\beta) .
\end{aligned}
$$

Taking the smallest possible $\beta$ (i.e., $\beta=0$ ) the second and the forth component of this expression will not increase. The value of the third component does not depend only on $\beta$; in fact, it depends on $\alpha+\beta$. For this reason, we can take $\beta=0$. Combining these facts, it yields that

$$
H_{2}(3) \geq-1-\frac{4}{3} \alpha(1-\alpha)-\frac{64}{3 \sqrt{6}} \alpha(1-\alpha) \text {. }
$$

The smallest value of the right hand side of this inequality is achieved for $\alpha=1 / 2$. In this case,

$$
H_{2}(3) \geq-1-\frac{1}{3}-\frac{16}{3 \sqrt{6}}=-\frac{4}{3}-\frac{8}{9} \sqrt{6}=-3.51 \ldots
$$

Combining two parts of the proof we obtain the conclusion of the theorem. Furthermore, the above shows that the extremal functions are

$$
\begin{aligned}
& f(z)=\frac{1}{2}\left[\frac{z}{(1+z)^{2}}+\frac{z}{1-2 z t_{0}+z^{2}}\right], \\
& f(z)=\frac{1}{2}\left[\frac{z}{(1-z)^{2}}+\frac{z}{1+2 z t_{0}+z^{2}}\right],
\end{aligned}
$$

where $t_{0}=1 / \sqrt{6}$.

\section{Bounds of $H_{2}(n), n \geq 4$, in $T$}

It is easily seen that $H_{2}(n) \leq n(n+2)$ for any typically real function. By Theorem 9, this estimate is sharp providing that $n$ is an odd integer. At the beginning of this section we will prove the following.

Theorem 13. For $T$ one has

$$
\min \left\{a_{n} a_{n+2}: f \in T\right\}=-1 \text {. }
$$

Proof. The coefficients of the series expansion of function $f \in$ $T$ can be written as follows:

$$
a_{n}=\int_{0}^{\pi} \frac{\sin (n \theta)}{\sin \theta} d \nu(\theta), \quad \nu \in P_{[0, \pi]} .
$$

Hence,

$$
\begin{aligned}
a_{n} a_{n+2}= & \int_{0}^{\pi} \frac{\sin ((n+1) \theta-\theta)}{\sin \theta} d \nu(\theta) \\
& \cdot \int_{0}^{\pi} \frac{\sin ((n+1) \theta+\theta)}{\sin \theta} d \nu(\theta) \\
= & \left(\int_{0}^{\pi} \frac{\sin (n+1) \theta}{\sin \theta} \cos \theta d \nu(\theta)\right)^{2} \\
& -\left(\int_{0}^{\pi} \cos (n+1) \theta d \nu(\theta)\right)^{2} .
\end{aligned}
$$

Since

$$
\int_{0}^{\pi} \cos (n+1) \theta d \nu(\theta) \leq \int_{0}^{\pi} d \nu(\theta)=1,
$$

we obtain

$$
a_{n} a_{n+2} \geq-1
$$

In order to prove that the estimate is sharp, let us take the measure $v$ for which support satisfies condition $(n+1) \theta=$ $\pi$. This measure corresponds to the function $f(z)=z /(1-$ $\left.2 z \cos (\pi /(n+1))+z^{2}\right)$.

Observe that $a_{n} a_{n+2}=-1$ holds not only for the measure stated above. Namely, the value -1 in (42) is taken also if $(n+$ 1) $\theta=k \pi$, where $k$ is any positive integer less than or equal to $n$. From this we conclude that the support of the measure has $n$ points $\theta_{k}=k \pi /(n+1)$ with weights $\alpha_{k}, k=1,2, \ldots, n$, such that $\sum_{k=1}^{n} \alpha_{k}=1$.

The weights satisfy

$$
\left(\sum_{k=1}^{n} \alpha_{k}(-1)^{k}\right)^{2}=1
$$

Indeed, if the support of $v$ consists of $n$ points then $f$ takes the form

$$
f(z)=\sum_{k=1}^{n} \alpha_{k} \frac{z}{1-2 z \cos \theta_{k}+z^{2}} .
$$

Using trigonometric identities we obtain

$$
\begin{aligned}
a_{n} & =\sum_{k=1}^{n} \alpha_{k}(-1)^{k+1}, \\
a_{n+2} & =\sum_{k=1}^{n} \alpha_{k}(-1)^{k},
\end{aligned}
$$

which results in (45).

Connecting (45) and $\sum_{k=1}^{n} \alpha_{k}=1$ we conclude that $f$ is of the form

$$
f(z)=\sum_{k=1, k \text { is odd }}^{n} \alpha_{k} \frac{z}{1-2 z \cos (k \pi /(n+1))+z^{2}}
$$


TABLE 1: The bounds of the Hankel determinants for functions defined by (51).

\begin{tabular}{lcc}
\hline Bounds of $H_{2}(n)$ & Equality in the lower bound for & Equality in the upper bound for \\
\hline$-9 \leq H_{2}(2) \leq 1$ & $t=1$ & $t=0$ \\
$-3.51 \ldots \leq H_{2}(3) \leq 15$ & $t=0,40 \ldots$ & $t=1$ \\
$-25 \leq H_{2}(4) \leq 4.46 \ldots$ & $t=1$ & $t=0.61 \ldots$ \\
$-7.84 \ldots \leq H_{2}(5) \leq 35$ & $t=0,72 \ldots$ & $t=1$ \\
$-49 \leq H_{2}(6) \leq 9.67 \ldots$ & $t=1$ & $t=0.79 \ldots$ \\
\hline
\end{tabular}

or

$$
f(z)=\sum_{k=1, k \text { is even }}^{n} \alpha_{k} \frac{z}{1-2 z \cos (k \pi /(n+1))+z^{2}}
$$

It means that for even $n$ the support of $\nu$ consists of $n / 2$ points, and for even $n$ the number of points of the support of $\nu$ is equal to $(n+1) / 2$ or $(n-1) / 2$.

Taking into account $\left|a_{n+1}\right| \leq n+1$ and Theorem 13, we obtain the following.

Theorem 14. For $T$ one has

$$
H_{2}(n) \geq-(n+1)^{2}-1 \text {. }
$$

Unfortunately, this bound is not sharp. However, the following can be conjectured.

Conjecture 15. For any positive integer $n$, the following estimate $\mathrm{H}_{2}(n) \geq-(n+1)^{2}$ holds. Moreover, this bound is sharp for even $n$.

This conjecture is supported by the facts that in the theorems concerning $\mathrm{H}_{2}(2)$ and $\mathrm{H}_{2}(3)$ the extremal functions are of the form

$$
\begin{aligned}
& f(z)=\frac{1}{2}\left[\frac{z}{(1+z)^{2}}+\frac{z}{1-2 z t+z^{2}}\right], \\
& f(z)=\frac{1}{2}\left[\frac{z}{(1-z)^{2}}+\frac{z}{1-2 z t+z^{2}}\right],
\end{aligned}
$$

for appropriately taken $t \in[-1,1]$. The exact bounds of the Hankel determinants for these functions are collected in Table 1 . They were obtained numerically.

\section{Remarks Concerning $H_{2}(n)$ in $T^{(2)}$}

In class $T$ we discuss subclass $T^{(2)}$ consisting of the functions which are odd. The definition of this class is

$$
T^{(2)}=\{f \in T: f(-z)=-f(z), z \in \Delta\} .
$$

For $f \in T^{(2)}$ the representation formula, similar to (2), is valid. Namely,

$$
f(z)=\int_{-1}^{1} \frac{z\left(1+z^{2}\right)}{\left(1+z^{2}\right)^{2}-4 z^{2} t^{2}} d \nu(t), \quad v \in P_{[-1,1]}
$$

Function $f$ has the Taylor series expansion

$$
f(z)=\sum_{n \text { is odd }} a_{n} z^{n}, \quad a_{n}=\int_{-1}^{1} U_{n-1}(t) d \nu(t) .
$$

The following inequalities are obvious:

$$
\begin{gathered}
-(n+1)^{2} \leq H_{2}(n) \leq 0 \quad \text { for even } n, \\
H_{2}(n) \leq(n+1)^{2}-1 \text { for odd } n ;
\end{gathered}
$$

equalities hold for $f(z)=z\left(1+z^{2}\right) /\left(1-z^{2}\right)^{2}$.

For a given class $A \subset \mathscr{A}$, let us denote by $\Psi_{n}(A), n \geq 1$, the set $\left\{\left(a_{n}, a_{n+2}\right): f \in A\right\}$. From (53) it follows that $\Psi_{n}(T)$ is the closed convex hull of the curve

$$
\lambda:[-1,1] \ni t \longrightarrow\left(U_{n-1}(t), U_{n+1}(t)\right) .
$$

From Theorem 13 and from the equivalence

$$
\left(a_{n}, a_{n+2}\right) \in \Psi_{n}(T) \Longleftrightarrow\left(a_{n}, a_{n+2}\right) \in \Psi_{n}\left(T^{(2)}\right),
$$

we get

$$
\min \left\{a_{n} a_{n+2}: f \in T^{(2)}\right\}=-1 .
$$

Hence, for odd $n$, we know that

$$
\min \left\{H_{2}(n): f \in T^{(2)}\right\} \geq-1 .
$$

The equality holds for functions (48) or (49) providing that $\alpha_{k}=\alpha_{n+1-k}$. Then, connecting the components of these formulae in pairs, we obtain

$$
\begin{aligned}
\alpha_{k} \frac{z}{1-} & 2 z \cos (k \pi /(n+1))+z^{2} \\
& +\alpha_{n+1-k} \frac{z}{1-2 z \cos ((n+1-k) \pi /(n+1))+z^{2}} \\
= & 2 \alpha_{k} \frac{z\left(1+z^{2}\right)}{\left(1+z^{2}\right)^{2}-4 z^{2} \cos ^{2}(k \pi /(n+1))} .
\end{aligned}
$$

With help of the argument given in the proof of Theorem 13, we eventually obtain the odd functions for which $a_{n} a_{n+2}=$ -1 .

\section{Conflict of Interests}

The author declares that there is no conflict of interests regarding the publication of this paper. 


\section{References}

[1] C. Pommerenke, "On the coefficients and Hankel determinants of univalent functions," Journal of the London Mathematical Society, vol. 41, pp. 111-122, 1966.

[2] C. Pommerenke, "On the Hankel determinants of univalent functions," Mathematika, vol. 14, pp. 108-112, 1967.

[3] A. Janteng, S. A. Halim, and M. Darus, "Coefficient inequality for a function whose derivative has a positive real part," Journal of Inequalities in Pure and Applied Mathematics, vol. 7, no. 2, article 50, 2006.

[4] A. Janteng, S. A. Halim, and M. Darus, "Hankel determinant for starlike and convex functions," International Journal of Mathematical Analysis, vol. 1, no. 13, pp. 619-625, 2007.

[5] S. K. Lee, V. Ravichandran, and S. Supramaniam, "Bounds for the second Hankel determinant of certain univalent functions," Journal of Inequalities and Applications, vol. 2013, article 281, 2013.

[6] D. Vamshee Krishna and T. Ramreddy, "Hankel determinant for starlike and convex functions of order alpha," Tbilisi Mathematical Journal, vol. 5, no. 1, pp. 65-76, 2012.

[7] C. Selvaraj and T. R. K. Kumar, "Second Hankel determinant for certain classes of analytic functions," International Journal of Applied Mathematics, vol. 28, no. 1, pp. 37-50, 2015.

[8] W. Ma and D. Minda, "A unified treatment of some special classes of univalent functions," in Proceedings of the Conference on Complex Analysis, Conference Proceedings and Lecture Notes in Analysis, Vol. 1, pp. 157-169, International Press, Tianjin, China, 1992.

[9] J. Sokół and J. Stankiewicz, "Radius of convexity of some subclasses of strongly starlike functions," Zeszyty Naukowe Politechniki Rzeszowskiej. Matematyka, vol. 19, pp. 101-105, 1996.

[10] M.-S. Liu, J.-F. Xu, and M. Yang, "Upper bound of second Hankel determinant for certain subclasses of analytic functions," Abstract and Applied Analysis, vol. 2014, Article ID 603180, 10 pages, 2014.

[11] A. K. Mishra and P. Gochhayat, "Second Hankel determinant for a class of analytic functions defined by fractional derivative," International Journal of Mathematics and Mathematical Sciences, vol. 2008, Article ID 153280, 10 pages, 2008.

[12] J. W. Noonan and D. K. Thomas, "On the Hankel determinants of areally mean $p$-valent functions," Proceedings of the London Mathematical Society, vol. 25, pp. 503-524, 1972.

[13] G. Singh, "Hankel determinant for a new subclass of analytic functions," Scientia Magna, vol. 8, no. 4, pp. 61-65, 2012.

[14] G. Singh, "Hankel determinant for analytic functions with respect to other points," Engineering Mathematics Letters, vol. 2, no. 2, pp. 115-123, 2013.

[15] T. Hayami and S. Owa, "Generalized hankel determinant for certain classes," International Journal of Mathematical Analysis, vol. 4, no. 52, pp. 2573-2585, 2010.

[16] K. O. Babalola, "On $\mathrm{H}_{3}(1)$ Hankel determinants for some classes of univalent functions," in Inequality Theory and Applications, vol. 6, 1.7, Nova Science Publishers, New York, NY, USA, 2010.

[17] G. Shanmugam, B. Adolf Stephen, and K. O. Babalola, "Third Hankel determinant for $\alpha$-starlike functions," Gulf Journal of Mathematics, vol. 2, no. 2, pp. 107-113, 2014.

[18] P. T. Mocanu, "Une proprit de convexit gnralise dans la thorie de la representation conforme," Mathematica (Cluj), vol. 11, no. 34, pp. 127-133, 1969.
[19] M. S. Robertson, "On the coefficients of a typically-real function," Bulletin of the American Mathematical Society, vol. 41, no. 8, pp. 565-572, 1935.

[20] D. J. Hallenbeck and T. H. MacGregor, Linear Problems and Convexity Techniques in Geometric Function Theory, Monographs and Studies in Mathematics 22, Pitman Adavanced Publishing Program, Boston, Mass, USA, 1984.

[21] W. Ma, "Generalized Zalcman conjecture for starlike and typically real functions," Journal of Mathematical Analysis and Applications, vol. 234, no. 1, pp. 328-339, 1999.

[22] W. Szapiel, Extremal problems for convex sets. Applications to holomorphic functions [Dissertation XXXVII], UMCS Press, Lublin, Poland, reviewer: J. Krzyż, 1986 (Polish).

[23] W. Szapiel, "Extreme points of convex sets (II). Inuence of normalization on integral representation," Bulletin de l'Académie Polonaise des Sciences, Série des Sciences Mathématiques, vol. 29, pp. 535-544, 1981. 


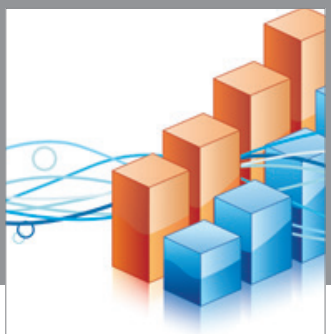

Advances in

Operations Research

vatem alat4

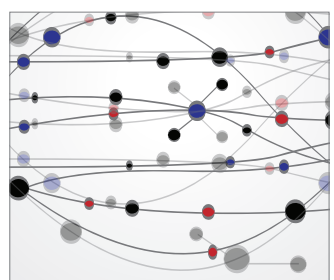

\section{The Scientific} World Journal
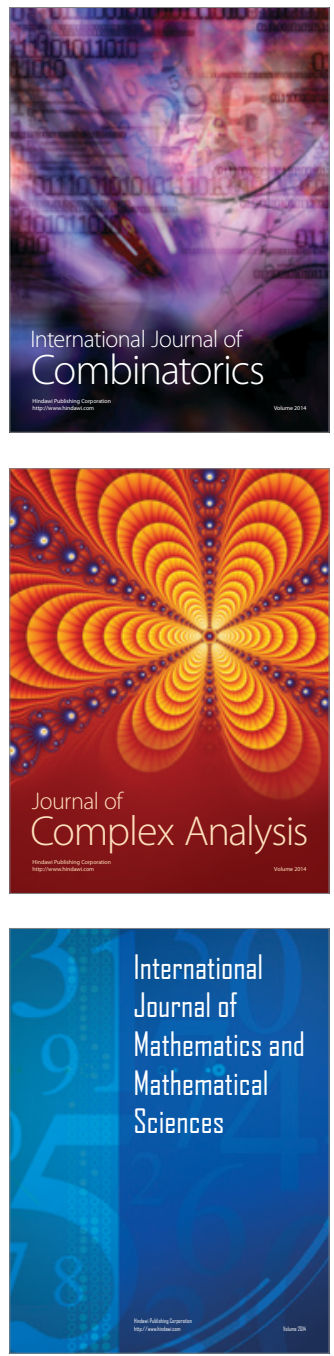
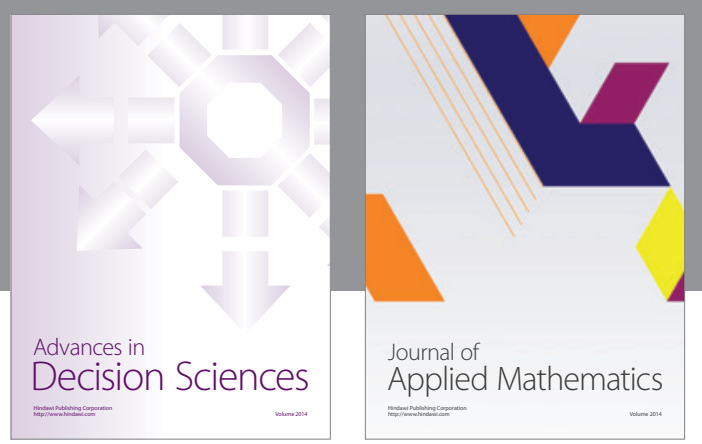

Algebra

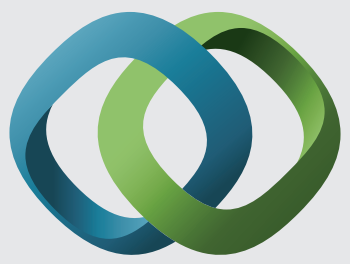

\section{Hindawi}

Submit your manuscripts at

http://www.hindawi.com
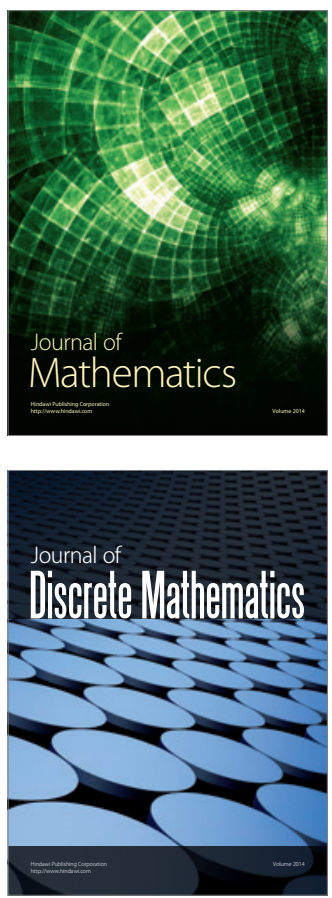

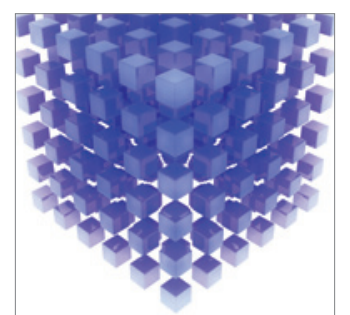

Mathematical Problems in Engineering
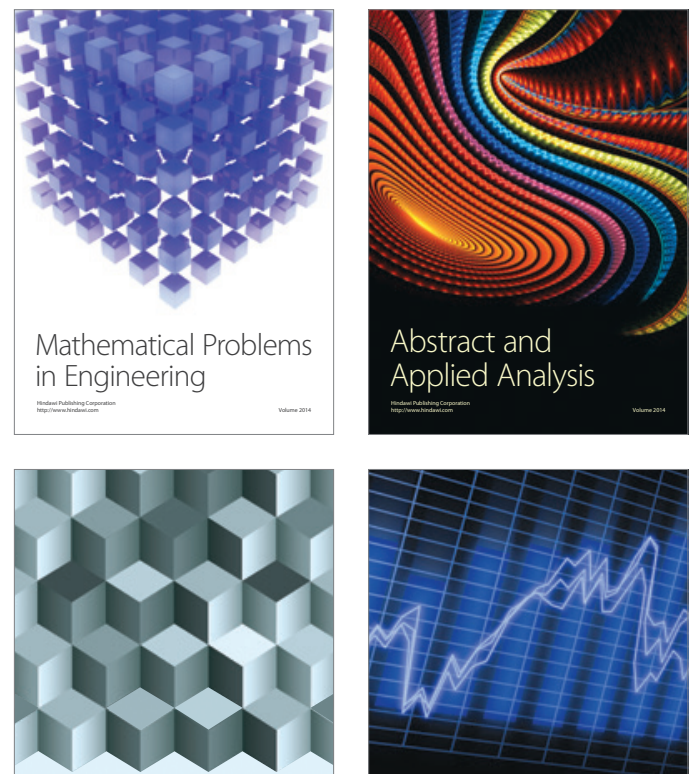

Journal of

Function Spaces

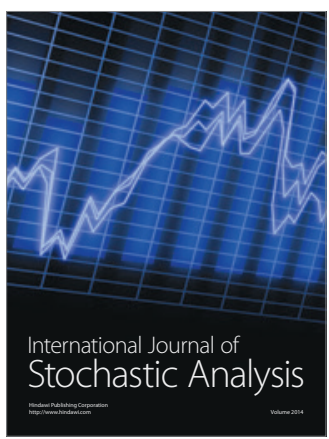

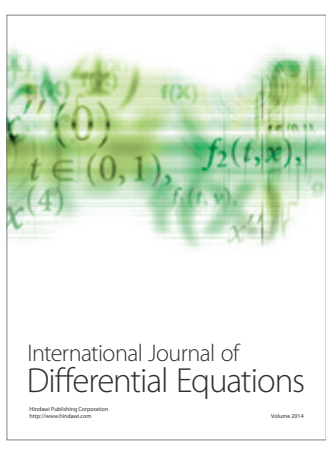
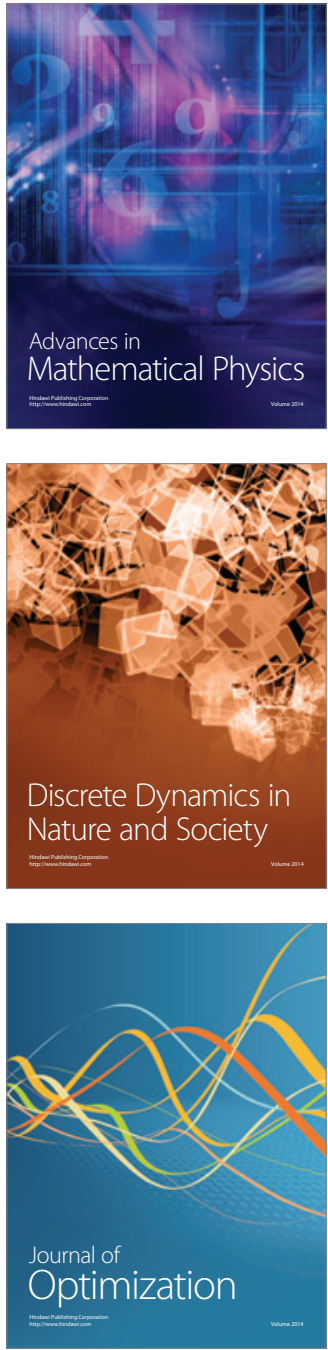